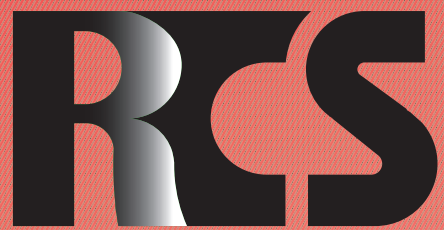

Depósito legal ppi $201502 Z U 4662$

Esta publicación científica en formato digital es continuidad de la revista impresa Depósito Legal: pp $197402 Z U 789$

- ISSN: 1315-9518 • ISSN-E: 2477-9431

Revista de Ciencias Sociales

Universidad del Zulia. Revista de la Facultad de Ciencias Económicas y Sociales Vol. XXVII. No. 2

Abril-Junio 2021

Esta publicación científica en formato digital es continuidad de la revista impresa Depósito Legal: pp $197402 Z$ Z789 ISSN: 1315-9518 


\title{
Situación de la mediana empresa ecuatoriana: Rasgos distintivos de sus prácticas gerenciales
}

\author{
Aguirre Benalcázar, Martha Cecilia" \\ Romero Hidalgo, Oscar Mauricio** \\ Jaramillo Paredes, Marcia Fabiola*** \\ Ruiz Carrillo, Jonathan Alexander ${ }^{* * * *}$
}

\section{Resumen}

La globalización de la economía, desarrollo de nuevos productos y los avances tecnológicos, han propiciado que las organizaciones requieran profesionales calificados para afrontar esos grandes retos. El objetivo de este artículo es analizar la situación de la mediana empresa ecuatoriana, destacando los rasgos distintivos de sus prácticas gerenciales como soporte de crecimiento. La metodología empleada es de tipo descriptiva, con un diseño de investigación no experimental, de campo, teniendo como población a las medianas empresas de 3 ciudades del Ecuador, activas en las páginas del Servicio de Rentas Internas en el año 2019. La técnica utilizada para la recolección de datos fue la encuesta, apoyada en un cuestionario de 15 ítems, aplicado a 50 gerentes y representantes principales de las unidades económicas. Los resultados demuestran que las medianas entidades trabajan arduamente para mantenerse operando en un mercado exigente, teniendo una dinámica de asuntos internos como principios, valores y clima organizacional que debe mejorarse, pues los aspectos financieros como el control y la evaluación son bien llevados. Se concluye, que deben apoyarse en la innovación y alianzas estratégicas para incrementar su productividad, comercialización, eficiencia y competitividad, mejorando sus resultados en el mercado nacional, con oportunidades de crédito y posibilidad de internacionalizarse.

Palabras clave: Medianas empresas; prácticas gerenciales; competitividad; desafíos; Ecuador.

Doctora en Ciencias Administrativas. Máster en Administración de Empresas. Profesora Titular de la Universidad Técnica de Machala, Ecuador. E-mail: maguirre@utmachala.edu.ec iD ORCID: https://orcid.org/0000-0002-61682899

** Doctor en Ciencias Administrativas. Magíster en Dirección de Empresas. Profesor Titular de la Universidad Técnica de Machala, Ecuador. E-mail: oromero@utmachala.edu.ec iD ORCID: https://orcid.org/0000-003-0018-5546

*** Magíster en Administración de Empresas. Profesora Titular de la Universidad Técnica de Machala, Ecuador. E-mail: mjaramillo@utmachala.edu.ec iD ORCID: https://orcid.org/0000-0002-0477-042X

***** Doctor en Ingeniería Industrial. Magíster en Docencia Universitaria e Investigación Educativa. Director de la Unidad de Investigación Desarrollo e Innovación Tecnológica del Instituto Superior Tecnológico El Oro - Machala, Ecuador. E-mail: jruiz@utmachala.edu.ec iD) ORCID: https://orcid.org/0000-0002-9183-0004 


\title{
Situation of the Ecuadorian medium- sized company: Distinctive features of its management practices
}

\begin{abstract}
The globalization of the economy, the development of new products and technological advances, have led organizations to require qualified professionals to face these great challenges. The objective of this article is to analyze the situation of the Ecuadorian medium-sized company, highlighting the distinctive features of its management practices as a support for growth. The methodology used is descriptive, with a nonexperimental, field research design, with the population of medium-sized companies from 3 cities in Ecuador, active on the pages of the Internal Revenue Service in 2019. The technique used to the data collection was the survey, supported by a questionnaire of 15 items, applied to 50 managers and main representatives of the economic units. The results show that medium-sized entities work hard to keep operating in a demanding market, having a dynamic of internal affairs such as principles, values and organizational climate that must be improved, since financial aspects such as control and evaluation are well managed. It is concluded that they must rely on innovation and strategic alliances to increase their productivity, commercialization, efficiency and competitiveness, improving their results in the national market, with credit opportunities and the possibility of internationalization.
\end{abstract}

Keyword: Medium businesses; managerial practices; competitiveness; challenges; Ecuador.

\section{Introducción}

La globalización de la economía, la apertura comercial, el desarrollo de nuevos productos, la formación de grupos con presencia internacional y los avances tecnológicos, han propiciado que las organizaciones requieran de profesionales altamente calificados, ocupando un papel estratégico dentro del negocio (García y Rodríguez, 2015), de manera de aprender, adaptarse y cambiar frente a un entorno empresarial marcado por la incertidumbre, que exige una gestión eficiente para afrontar grandes retos.

Por lo que, participar actualmente en el mercado, conlleva un costo sobresaliente que no es otro que la práctica gerencial. Siendo para García y Rodríguez (2015), un tema que reviste una predominancia relativamente reciente, con impacto positivo para el crecimiento económico de los países y la competitividad desde las empresas, pues todas las acciones que la gerencia lleve a cabo en ámbitos internos y externos de la organización, para dar cumplimiento a los objetivos propuestos, permitirá garantizar el éxito.

En el caso de Ecuador, en los últimos años se han realizado transformaciones en lo político, económico y social, a través de políticas propias del desarrollo tradicional de la economía de mercado, perfilando la distribución de la riqueza de manera más equitativa en la sociedad (Rodríguez-Mendoza y Aviles-Sotomayor, 2020). Teniendo las empresas la oportunidad de producir, generar empleos y crear tejido empresarial con peso en el Producto Interno Bruto (PIB), siendo una tarea factible para las pequeñas y medianas empresas (pymes).

Estas representan el $95 \%$ de las unidades productivas ecuatorianas, con una participación importante en el sector de la producción y el comercio (Rodríguez-Mendoza y Aviles-Sotomayor, 2020), donde el capital 
es proporcionado por una o dos personas que establecen una "sociedad", los propios dueños dirigen la marcha del negocio, su número de trabajadores va de 16 hasta 250 personas, su administración es mayoritariamente empírica, abastecen un mercado amplio y están en proceso de crecimiento, dado que la pequeña tiende a ser mediana y aspira a ser grande de acuerdo a Peña y Vega (2017).

En cuanto a la distribución de las pymes por ubicación geográfica, puede destacarse que la mayor cantidad se encuentra en las provincias de Quito y Guayaquil, quizás por la concentración de líneas de microcrédito que según Peña y Vega (2017), el 73,88\% de microcréditos de bancos privados se desembolsaron en las dos provincias. En este sentido, son empresas que han adquirido relevancia con los años y son el soporte del aparato productivo del país, evidenciado en su sustancial contribución en la generación de producto, empleo e ingreso, que las convierte en pilar de bienestar.

Entre los principales logros de la mediana empresa específicamente, constituida por 13.693 establecimientos, se denota su aporte en la generación de fuentes de trabajo (14\%) y de ingresos para la economía (18\%) (DSG Consulting, 2020). Su ventaja frente a empresas de mayor envergadura, radica en su adaptabilidad a los requerimientos del mercado, pues tienen muchos años operando; y la respuesta efectiva a las necesidades de los clientes, agrupando unidades económicas con un nivel de desarrollo sólido (Ortiz, et al., 2019).

No obstante, existen barreras que frenan el surgimiento y desarrollo de estas organizaciones en el contexto nacional, como el restringido acceso al crédito, lo que propicia un ciclo de limitaciones que le impiden emprender procesos de mejora continua $\mathrm{y}$ poder enfrentar la competencia del mercado en mejores condiciones (Padilla, Lascano y Jiménez, 2018). A pesar de los esfuerzos de la banca pública y privada, los procesos para el crédito siguen siendo muy engorrosos, complicados y demorados. Asimismo, sufren de asimetrías de información que le impiden competir en igualdad de condiciones con grandes empresas (Corporación Financiera Nacional [CFN], 2019).

Además, de la ausencia de programas de certificación laboral dirigidos a mejorar la calidad de empleo, falta de capacitación y asistencia técnica, así como la carencia de políticas de liberalización de mercados, le impiden su introducción en la economía internacional, pues casi todas se enfocan en lo local o nacional y son pocas las que exportan, con una trascendencia que apenas llega a Colombia o Perú (Peña y Vega, 2017; Arguello, 2019). Contexto desafiante que incide en la eficiencia, dinamismo y soporte institucional operacional de las unidades económicas.

Bajo estos planteamientos, se consideró pertinente analizar la situación de la mediana empresa ecuatoriana, destacando los rasgos distintivos de sus prácticas gerenciales, a través de la revisión de asuntos internos a las unidades económicas: Principios corporativos, valores éticos, cumplimiento de la visión, desempeño, comunicación, clima organizacional, control y evaluación interna; así como externos: Desafíos, competitividad y comercialización.

\section{Metodología}

El enfoque epistemológico utilizado fue el empírico - inductivo, con un diseño de investigación no experimental, de campo y un tipo de estudio descriptivo. La población objeto de análisis, estuvo conformada por medianas empresas de tres ciudades principales del Ecuador; consideradas activas en el Servicio de Rentas Internas para el año 2019 y cuyo margen de utilidad haya sido superior a los $\$ 100.000$ en el año. Cumpliendo con estos criterios se encontraron 50 unidades económicas.

Como técnica para la recolección de datos se utilizó la encuesta, mediante el uso de un cuestionario compuesto por 15 items, aplicado a los gerentes y representantes principales de las medianas empresas, orientado a recolectar información general 
de las entidades, asuntos internos (principios corporativos, valores éticos, cumplimiento de la visión, desempeño, comunicación, clima organizacional, control y evaluación interna) y externos (desafíos, competitividad y comercialización).

Para el procesamiento de los datos recolectados del cuestionario, se utilizó el programa estadístico SPSS (Statistical Package for the Social Sciences) Versión 23 y la Hoja de Cálculo Excel, que permitieron obtener los resultados necesarios para el análisis del estudio. Entre los distintos procedimientos para el análisis, se encuentran: Distribución de los datos, valores o puntuaciones obtenidas para cada item; distribución de frecuencias (absolutas y relativas), que permitieran generar los gráficos; y presentación de los datos de manera escrita y tabulada.

Debe destacarse que el cuestionario aplicado, contuvo preguntas de selección simple, por lo que la etapa de inferencia estadística toma aquellos resultados de una mayor frecuencia como un rasgo predominante en la población, y el número de opciones promedio marcado para de cada ítem determina la cantidad de factores involucrados para los aspectos analizados.

\section{Resultados y discusión}

Como principales resultados de la investigación se encuentran, unidades empresariales que pese a la adversidad de la economía del Ecuador, siguen trabajando arduamente para incrementar sus niveles de productividad, procesos tecnológicos y crecimiento estructural, con prácticas gerenciales que dan cuenta de su avance y de algunos retrasos en asuntos internos y externos que son necesarios mejorar.

En relación a los principios corporativos de estas empresas, se denota su compromiso ciudadano $(30,20 \%)$, como aspecto clave para operar; seguido del compromiso ético $(23,10 \%)$ y con el medio ambiente $(19,90 \%)$. En menor proporción, las empresas se inclinan a aspectos socio-culturales y al respeto por la diversidad y multiculturalidad (ver Gráfico I). en ese sentido, Daniel, et al. (2019), indican que en las organizaciones es necesario mantener principios sociales como praxis empresarial, pero muchas no terminan de considerarlos como elementales y han fijado otros.

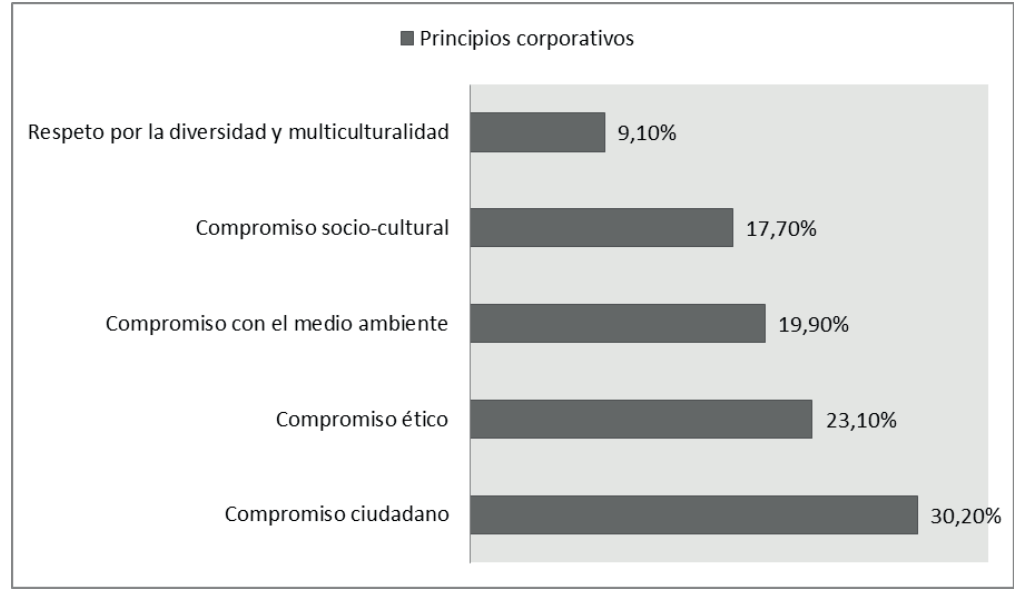

Fuente: Elaboración propia, 2020.

Gráfico I: Principios corporativos de las medianas empresas 
En el caso de estas entidades, se observa un apoyo significativo por el compromiso ciudadano y ético, pero es importante se incremente el aspecto socio-cultural, pues las empresas se deben a un entorno que muchas veces presenta problemas y requiere de recursos que permitan contribuir a su solución o a la mejora de la sociedad. Siendo para Wang, et al. (2016), una estrategia social necesaria, voluntaria y con creación de valor.

Con respecto a los valores éticos, las medianas empresas declaran soportarse en la honestidad $(35,60 \%)$, respeto $(33,30 \%)$, justicia $(30,20 \%)$, libertad $(23,10 \%)$ y responsabilidad $(19,90 \%)$, como valores clave en cada una de sus actividades (ver Gráfico II). Para Velásquez y Núñez (2009), a pesar de que ninguna organización es igual a otra, es posible encontrar elementos comunes que las coadyuven al éxito, manifestándose valores de diferentes formas que definen su cultura organizacional y guían la práctica gerencial.

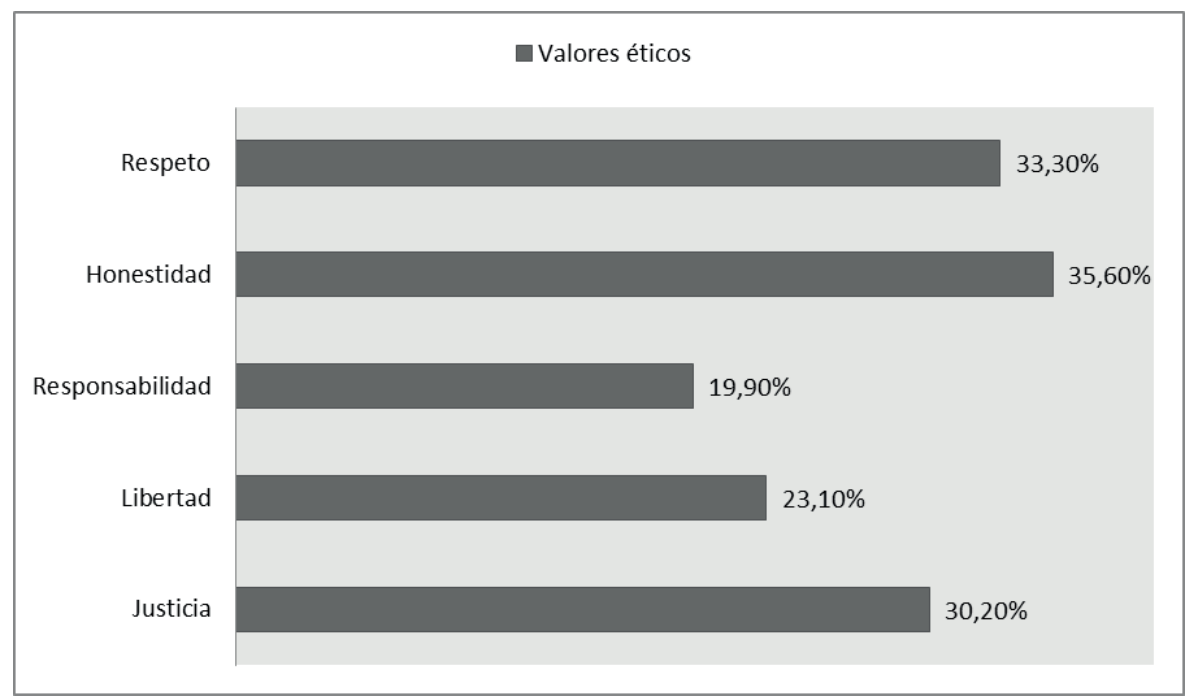

Fuente: Elaboración propia, 2020.

\section{Gráfico II: Valores éticos de las medianas empresas}

En cuanto a los elementos que mayormente se aplican para cumplir la visión de las medianas empresas, se tiene que el $35,60 \%$ de estas, optimizan los recursos de la mejor forma, un $33 \%$ cumple la normativa en los procesos y el $25 \%$ se aboca a rendir en sus actividades lo más que puedan (ver Gráfico III). Siendo elementos necesarios en toda unidad económica de acuerdo a Thompson, et al. (2012), que se suman a otros para complementar las estrategias principales de las empresas. 


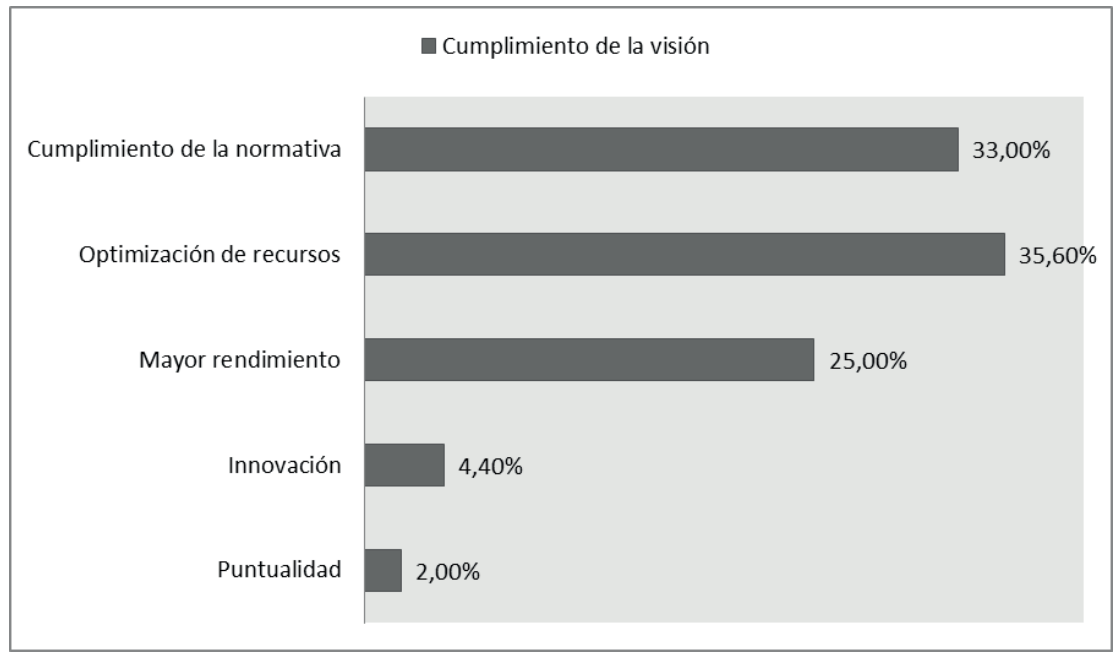

Fuente: Elaboración propia, 2020.

\section{Gráfico III: Cumplimiento de la visión en las medianas empresas}

No obstante, siguiendo a Romero y Zabala (2018), muy pocas empresas están considerando la innovación como elemento estratégico esencial, que proporciona una perspectiva respecto a la dirección a largo plazo, sirviendo de guía en el quehacer gerencial. Esta variable se ha convertido en eje transversal para todas las actividades organizacionales, siendo pilar fundamental en su crecimiento y desarrollo, pues mantiene el norte de la misión que se acompaña de los objetivos propuestos (Fuenmayor, Dadul y Gutiérrez, 2017; Navarro-Caballero, et al., 2020).

Ahora bien, las unidades económicas encuestadas afirman que las habilidades que predominan para su desempeño, supervisadas constantemente por los gerentes, son la colaboración $(36,60 \%)$, creatividad $(27,60 \%)$, entusiasmo $(20,15 \%)$ y dinamismo $(15,30 \%)$, tal como se observan en el Gráfico IV. A criterio de Thompson, et al. (2012), todas las habilidades con las que cuente la empresa en pro de mejorar sus procesos administrativos - productivos deben cuidarse y mantenerse en el tiempo, que implicarán una supervisión seguida. Siendo oportuno recompensar a los colaboradores con alto desempeño, que generará efecto en otros que quieran también sobresalir para ser recompensados. 


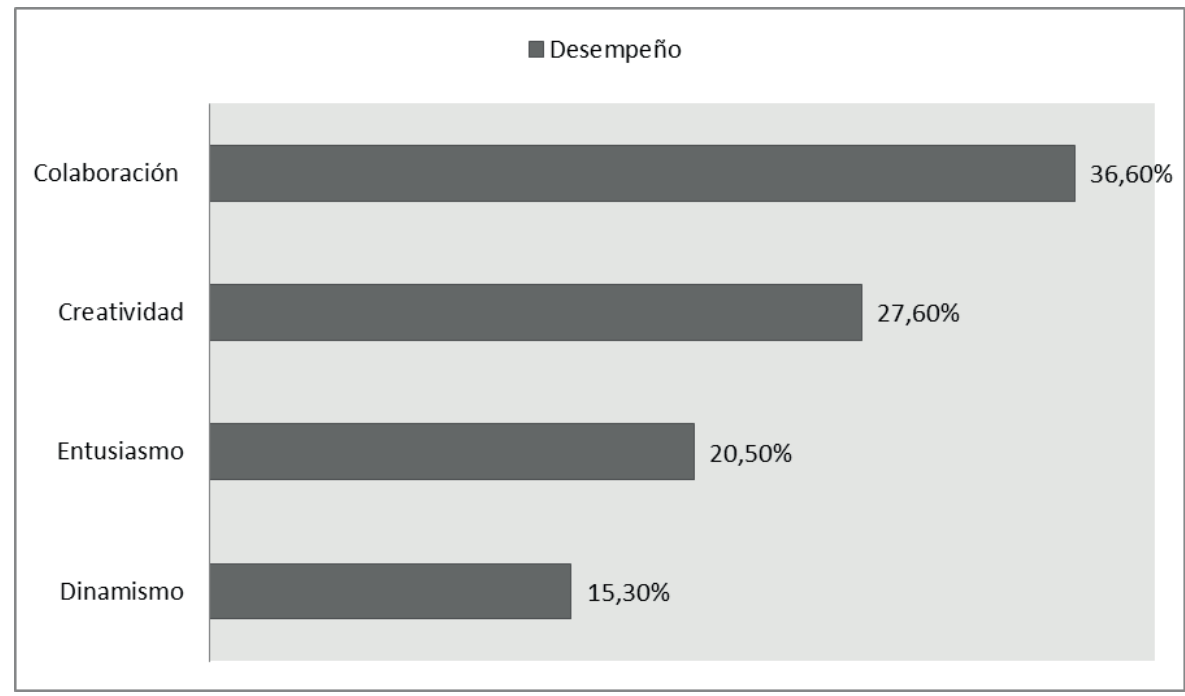

Fuente: Elaboración propia, 2020.

\section{Gráfico IV: Desempeño en las medianas empresas}

Esta visión de desempeño es vista por Pedraza, Amaya y Conde (2010), como el valor que se aporta a los diferentes episodios conductuales de los individuos que contribuyen en la eficiencia organizacional, por lo que, debe prestársele especial atención dentro del proceso de administración de recursos humanos. En este sentido, Sanín y Salanova (2013) recomiendan que la evaluación de dicho desempeño debe proporcionar beneficios a la organización y a las personas, garantizando el alcance de los objetivos institucionales.

En relación a los medios de comunicación que utilizan las empresas con mayor frecuencia, sobresale el correo electrónico $(33,50 \%)$, que ha sido una vía práctica y rápida devenida con la era del internet, implementándose en muchas organizaciones a nivel global, sustituyendo encuentros presenciales. Otro grupo de empresas, utiliza las reuniones $(30,40 \%)$ y llamadas telefónicas $(22,20 \%)$, que son medios comunicativos tradicionales y hasta interactivos entre el personal, dado que el contacto es directo, pudiendo aclarar de manera inmediata cualquier inquietud (ver Gráfico V). 


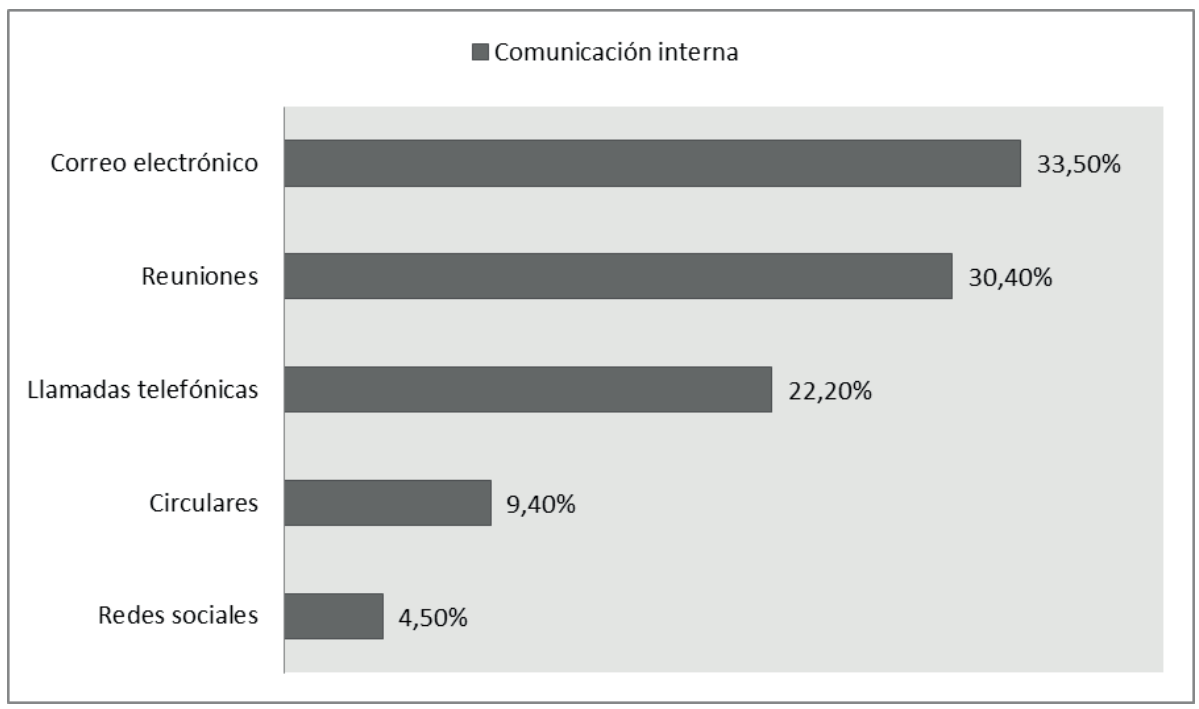

Fuente: Elaboración propia, 2020.

\section{Gráfico V: Medios de comunicación interna en las medianas empresas}

Al respecto, Pérez (2014) señala que la comunicación no debe descuidarse, debido a que representa una herramienta para dar respuesta a los continuos cambios en las empresas, que facilita la introducción de nuevos valores, políticas, pautas o procesos, así como la alineación de los empleados con los objetivos empresariales. En otras palabras, es un medio de cambio, que aumenta la satisfacción entre el personal y la rentabilidad financiera, que debe ser bien ejercida por los directivos.

Los gerentes y representantes encuestados, por otra parte, afirman que los factores que han cuidado para mejorar el clima organizacional, son el trabajo en equipo $(25,10 \%)$, relaciones interpersonales $(22,30 \%)$ y la comunicación $(17,50 \%)$. Seguido de la remuneración e incentivos (14,50\%), el liderazgo $(10,20 \%)$ y las condiciones físicas de trabajo (10,40\%) (ver Gráfico VI). Autores como Segredo, et al. (2017), exponen que los gerentes deben velar por un clima favorable para toda la empresa, donde destaquen las condiciones físicas de trabajo, las herramientas para todos los procesos y una buena remuneración, y en el caso de estas entidades ecuatorianas, el espacio físico es poco considerado, pudiendo repercutir en el desempeño del recurso humano.

Ciertamente, las relaciones interpersonales, el sostenido trabajo en equipo y una asertiva comunicación, traen resultados positivos para las organizaciones, no obstante, deben mantenerse unas condiciones físicas laborales aceptables, que propicien la productividad, de lo contrario, se generará malestar, estrés, desmotivación, falta de compromiso y la afectación de las relaciones entre empleados (Montiel y Díaz, 2018). 
Aguirre Benalcázar, Martha Cecilia; Romero Hidalgo, Oscar Mauricio; Jaramillo Paredes, Marcia Fabiola y Ruiz Carrillo, Jonathan Alexander

Situación de la mediana empresa ecuatoriana: Rasgos distintivos de sus prácticas gerenciales

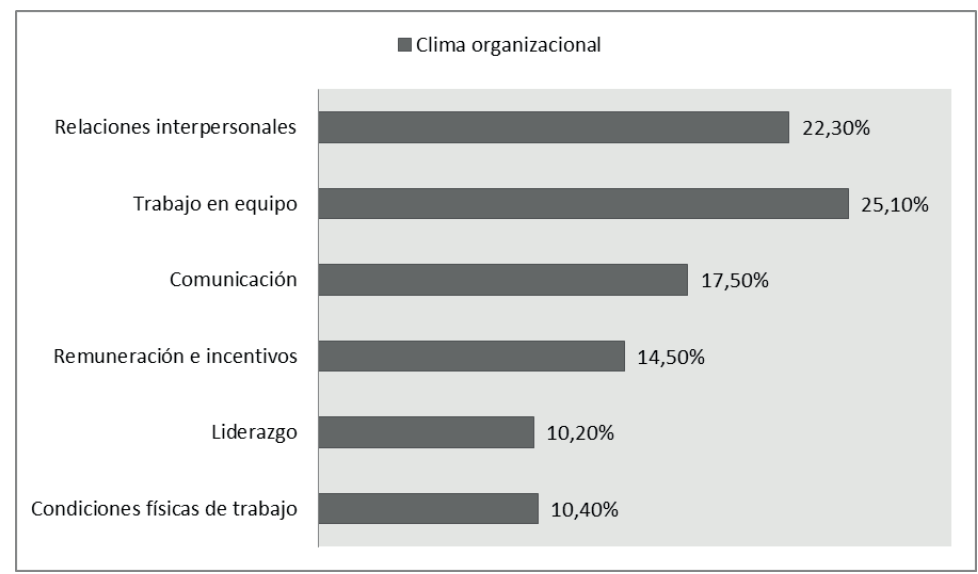

Fuente: Elaboración propia, 2020.

\section{Gráfico VI: Clima organizacional en las medianas empresas}

En cuanto a los instrumentos de control interno, que siguen estas organizaciones para el desarrollo de sus actividades, el $40,20 \%$ indica que se guían principalmente del reglamento interno, el 30,60\% utiliza el manual de funciones y un $16,80 \%$ las políticas internas. Escasas empresas declinan por las normas de seguridad ocupacional y el código de ética, como instrumentos fundamentales (ver Gráfico VII). En ese sentido, Mendoza y Bayón (2019) recalcan que todo conjunto de leyes y normas aplicables a una entidad, permitirán lograr la eficacia y eficiencia de las operaciones, mediante el uso adecuado de la contabilidad y la información financiera.

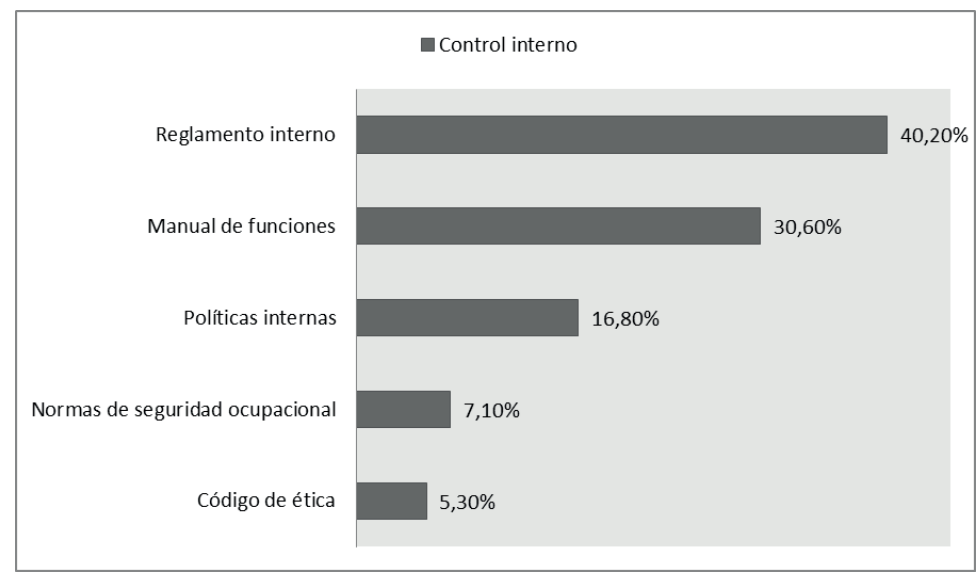

Fuente: Elaboración propia, 2020.

\section{Gráfico VII: Control interno en las medianas empresas}


Las mismas autoras Mendoza y Bayón (2019), señalan que, ante las condiciones en la que opera la economía ecuatoriana, con mayor grado de apertura externa y exigencias de la competencia internacional, el control oportuno y eficaz de la actividad económica, minimiza los problemas que enfrentan los directivos por no aplicar las normativas que regulan su actividad, donde las nuevas tecnologías de la información y las comunicaciones desempeñan un papel relevante. De esta manera, las medianas empresas analizadas, están tratando de cumplir con este aspecto, para no asumir riesgos basados en problemas de manejo, desperdicios, procesos productivos, comercialización o administración de costos.

De igual forma, el $58,50 \%$ de las empresas manifiestan que emplean las auditorías como mecanismo de evaluación interna. Siendo una herramienta mayormente utilizada por las entidades empresariales, donde una observación oportuna puede ayudar a revertir problemas financieros según Rodríguez (2020). El 36,50\% utiliza sistemas de control interno, también útil para procesos de contabilidad financiera y presentación de informes. A penas un $3 \%$ de las unidades económicas emplea comisiones de evaluación, tal como se aprecia en el Gráfico VIII.

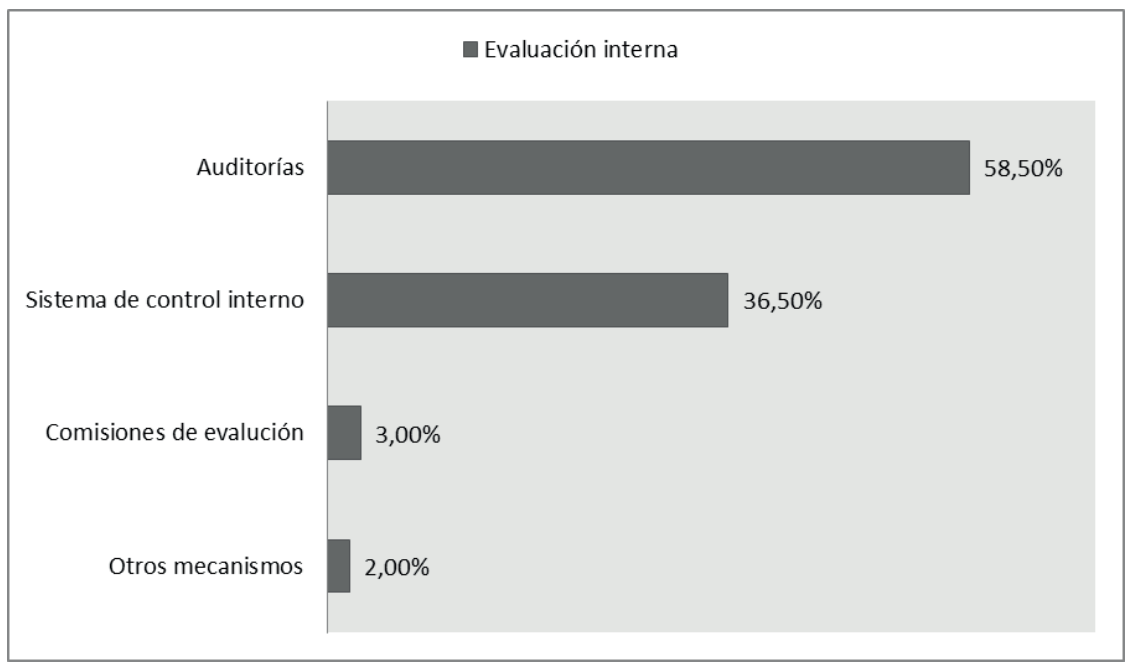

Fuente: Elaboración propia, 2020.

\section{Gráfico VIII: Evaluación interna en las medianas empresas}

Romero, et al. (2020), plantean que los gerentes deben estar atentos a la gestión financiera de las empresas, pues muchas veces fracasan por su mala administración o bajos niveles de alfabetización y disciplina financiera, siendo importante que empleen un sistema de contabilidad adecuado que pueda ser fácilmente monitoreado. En el mismo orden, Rodríguez (2020) expone que una apropiada labor de auditoría, mejorará y fortalecerá la situación de los negocios, donde la preparación de proyecciones de flujo de caja, junto con una buena documentación y un plan de negocios convincente, facilitará el 
acceso a créditos.

Por otra parte, las medianas entidades manifiestan que sus desafíos vienen dados por las acciones para superar la crisis $(34,50 \%)$, la toma de decisiones arriesgadas $(23,10 \%)$, las negociaciones que deben hacer $(22,10 \%)$ y la expansión de la empresa (20,30\%) (ver Gráfico IX). Tema que alertan cada día a los gerentes, ante los embates de la economía nacional y la dinámica de los sectores en los que operan. Rodríguez-Mendoza y Aviles-Sotomayor (2020), consideran que los desafíos que enfrentan las pymes en general son arduos, su evolución estructural y funcional no presenta avances por las dimensiones y exigencias del mercado, que coloca en juego el cierre de numerosas organizaciones.

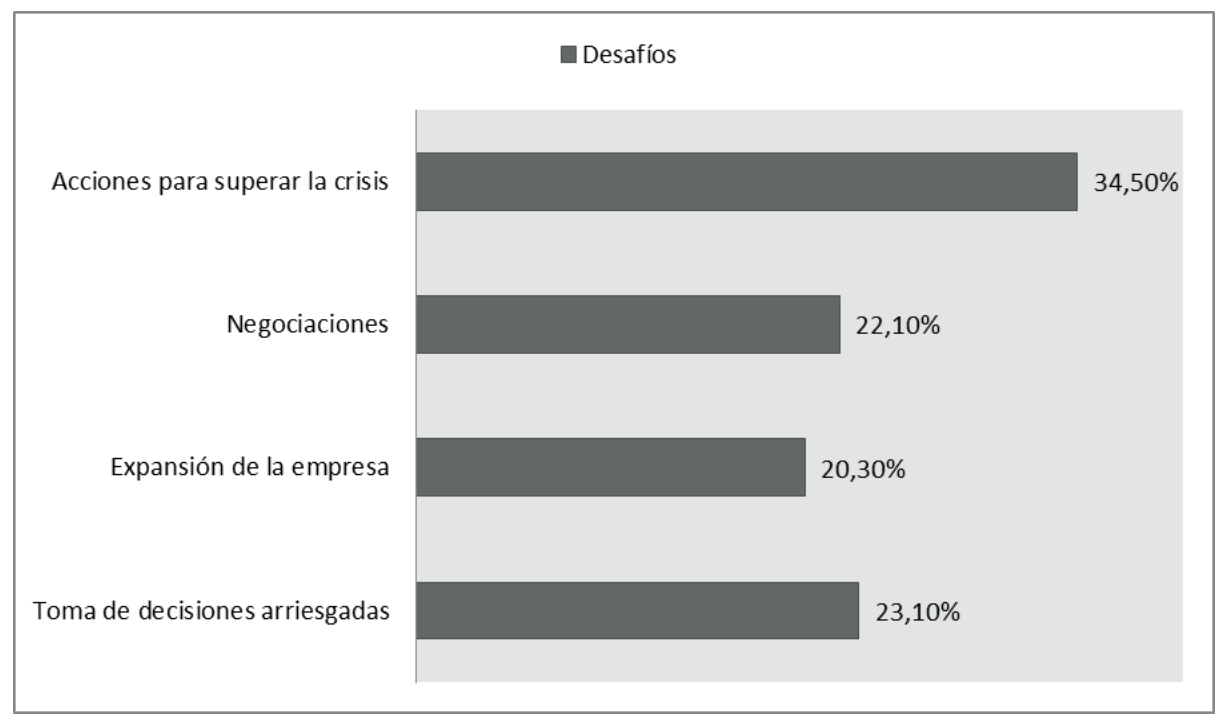

Fuente: Elaboración propia, 2020.

\section{Gráfico IX: Desafíos de las medianas empresas}

Ante la situación descrita, la competitividad es crucial, constituyendo un factor para la consolidación de sus planes en el mercado, su productividad y hasta eficiencia. De este modo, las empresas se han abocado a trabajar por la calidad del producto o servicio $(68,20 \%)$ (ver Gráfico X), que a criterio de Navarro, et al. (2020), es un aspecto a favor, dado que la mayoría ha creado nuevos bienes o servicios, o bien ha mejorado sus características, materiales y demás aspectos funcionales, pudiendo incrementarse las ventas. 


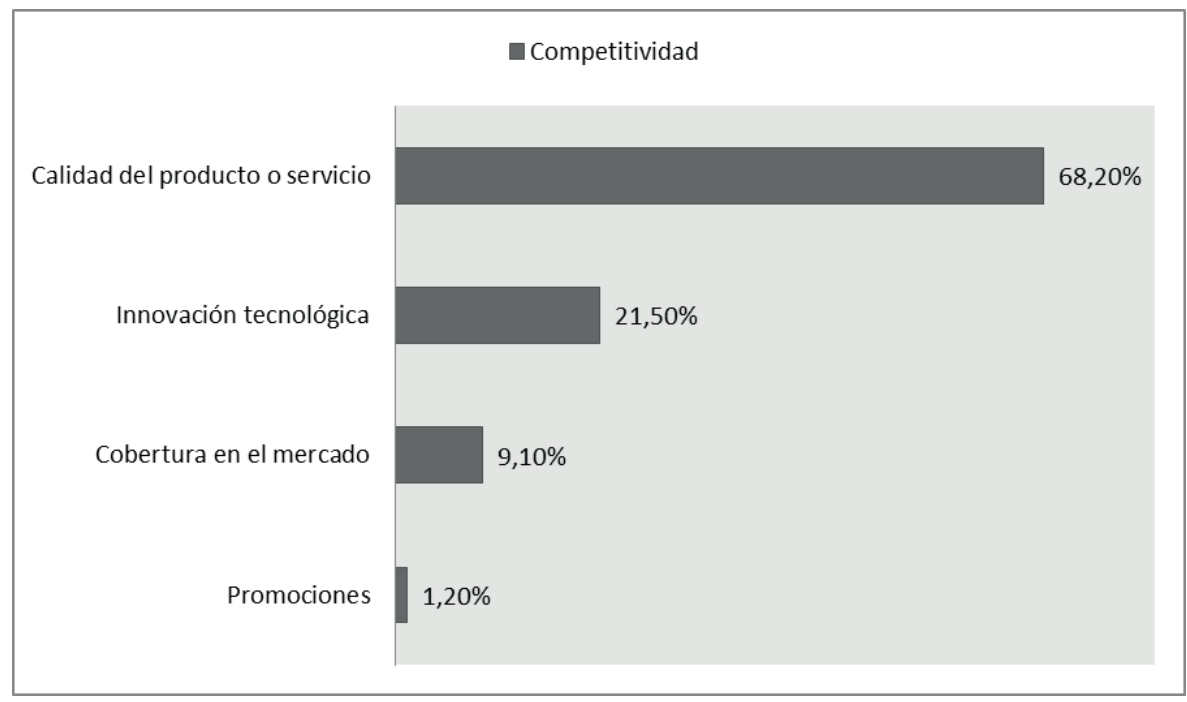

Fuente: Elaboración propia, 2020.

\section{Gráfico X: Competitividad en las medianas empresas}

Otras empresas declinan por la innovación tecnológica $(21,50 \%)$, variable clave que debiera impulsarse, puesto que constituye la causa directa de la competitividad y fuente de los cambios estructurales necesarios para el desarrollo empresarial (Odremán, 2014). Muy pocas unidades trabajan por la cobertura en el mercado $(9,10 \%)$ y las promociones $(1,20 \%)$, aspectos interesantes que también propician la competitividad.

Por último, el $62,50 \%$ de los gerentes y representantes encuestados informaron que las unidades económicas han diseñado planes de marketing para mejorar el proceso de comercialización. El 15,20\% recalcó que están tratando de hacer cambios en sus procesos productivos, mientras que el $12,30 \%$ ha introducido nuevos productos en el mercado, el resto afirma trabajar en mejoras para la distribución de bienes y servicios (ver Gráfico $\mathrm{XI})$. 


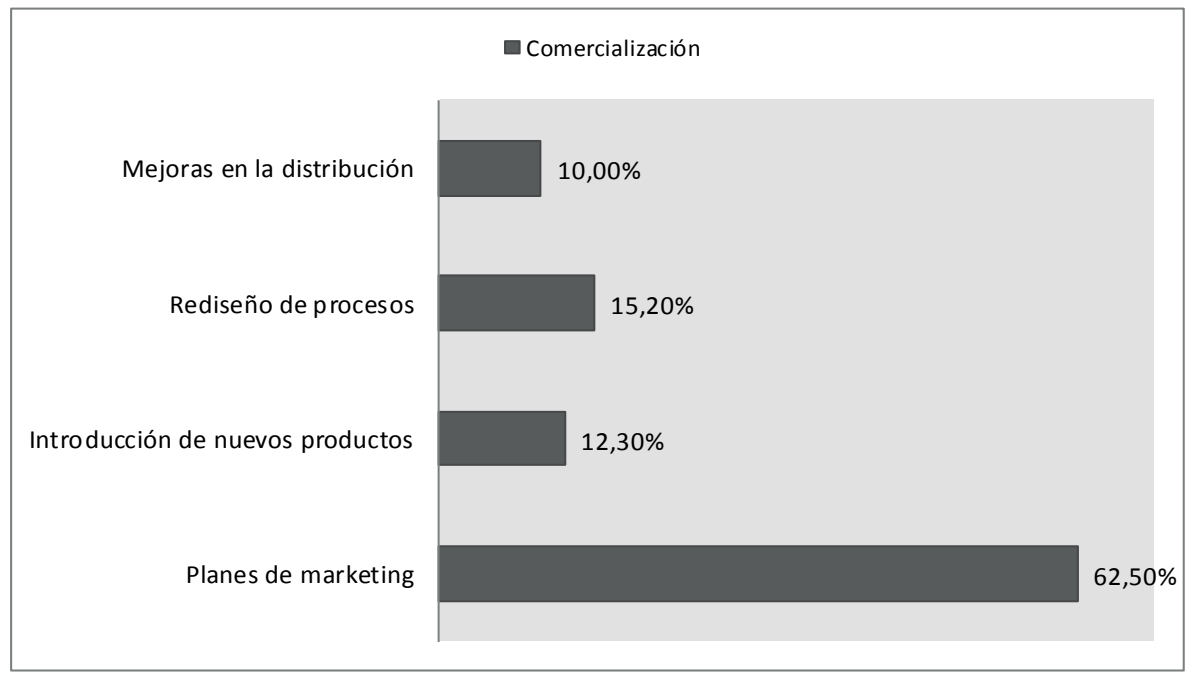

Fuente: Elaboración propia, 2020.

\section{Gráfico XI: Comercialización de las medianas empresas}

Estos elementos mencionados, se han hecho necesarios para dinamizar la comercialización de los bienes y servicios, pero no deben manejarse por aislados, convienen integrarse, haciendo uso de la innovación y la competitividad, para posicionarse en el mercado y consolidar los objetivos comerciales que beneficien a las empresas, hasta el punto de lograr su internacionalización, aprovechando las políticas de apertura que ha seguido Ecuador (Sotomayor, Capa y Ochoa, 2018; Quiñónez, et al., 2020). En otras palabras, deben implementarse mecanismos de gestión comercial apropiados, que permitan aprovechar las ventajas comparativas y competitivas de estas empresas y sus productos, para así impulsar su participación en el mercado internacional.

\section{Conclusiones}

Puede concluirse que estas medianas empresas ecuatorianas deben aprovechar las fortalezas que tienen en cuanto tamaño, estructura, sector donde operan, principios y valores organizacionales, que sumado a otros elementos pueden contrarrestar los problemas que enfrentan, ligados al financiamiento, cumplimiento pleno de la visión, clima organizacional, toma de decisiones arriesgadas, cobertura del mercado y comercialización de bienes y servicios, además de factores macroeconómicos que las coloca en una fuerte encrucijada.

Sin embargo, estas unidades empresariales han demostrado sobrevivir a esos embates, manteniéndose de pie, con un desempeño aceptable y medidas de control así como evaluación efectiva, que denotan prácticas gerenciales positivas. Siendo importante, que se apoyen en la innovación como elemento dinamizador de múltiples procesos que las beneficiaría, incrementando su productividad, eficiencia y competitividad, dando paso a consolidar sus objetivos en 
el mercado nacional, con oportunidades de crédito y la posibilidad de internacionalizarse.

Además, deben crearse planes donde su personal desarrolle actividades internas, que permitan el crecimiento sostenido y alianzas estratégicas entre empresas, o con otras de mayor tamaño como nuevas vías de asociación solidaria que potencien sus estructuras, gestión económica, capacidades y competencias, con el fin de poder operar en mejores condiciones, adaptándose a las exigencias de un entorno empresarial marcado por la incertidumbre.

\section{Referencias bibliográficas}

Arguello, A. (8 de enero de 2019). Un análisis de las MIPYMES en Ecuador. MarketingActivo. https:// marketingactivo.com/un-analisis-delas-mipymes-en-ecuador/2019/01/08/

Corporación Financiera Nacional - CFN (28 de agosto de 2019). CFN y CAF impulsan el crecimiento de las Pyme en Ecuador. CAF. Banco de Desarrollo de América Latina. https://www.caf. com/es/actualidad/noticias/2019/08/ cfn-y-caf-impulsan-el-crecimiento-delas-pyme-en-ecuador/

Daniel, O., Alvarado-Peña, L. J., Sansores, E. A., y Navarrete, J. E. (2019). Responsabilidad Social Empresaria: Hacia la conformación de una tipología de definiciones. Revista Venezolana de Gerencia, 24(85), 281-299.

DSG Consulting (2020). Características de las MiPymes en Ecuador [Mensaje en un blog]. https://www.dsg.ec/gestionempresas/mipymes-en-ecuador/

Fuenmayor, A., Dadul, Y., y Gutiérrez, J. M. (2017). Gestión tecnológica de la Facultad de Ciencias Económicas y Sociales de la Universidad del Zulia. Revista de la Universidad del Zulia, 8(20), 83-96.
García, M., y Rodríguez, M. A. (2015). Evaluación de las prácticas gerenciales. Negotium, 10(30), 142-162.

Mendoza, M. F., y Bayón, M. L. (2019). El control interno y las pymes. Sinapsis, 11(2), 19-30.

Montiel, P., y Díaz, Á. (2018). Clima laboral en el rendimiento de los empleados de las Pymes en el Ecuador. Journal of Science and Research: Revista Ciencia e Investigación, 3(11), 60-63.

Navarro-Caballero, M., Hernández-Fernández, L., Navarro-Manotas, E., y HernándezChacín, J. (2020). Innovación en las micro, pequeñas y medianas empresas familiares del sector manufacturero del Atlántico-Colombia. Revista de Ciencias Sociales (Ve), XXVI(4), 124144. https://doi.org/10.31876/rcs. $\underline{\mathrm{v} 26 \mathrm{i} 4.34653}$

Odremán, J. G. (2014). Gestión tecnológica: Estrategias de innovación y transferencia de tecnología en la industria. Universidad, Ciencia $y$ Tecnología, 18(73), 181-191.

Ortiz, K., Campos, E., Alvarado, M., y Alcívar, C. (2019). Análisis de las empresas familiares en Ecuador desde una óptica multivariante. Revista de Ciencias Sociales (Ve), 25(4), 161-174.

Padilla, M. P., Lascano, L. F., y Jiménez, W. R. (2018). La dinámica empresarial y el emprendimiento, factores determinantes para el desarrollo del ciclo de vida de las pymes. Revista Publicando, 5(15(2)), 308-325.

Pedraza, E., Amaya, G., y Conde, M. (2010). Desempeño laboral y estabilidad del personal administrativo contratado de la Facultad de Medicina de la Universidad del Zulia. Revista de Ciencias Sociales (Ve), XVI(3), 493505.

Peña, M. J., y Vega, N. E. (2017). Estructura de las PyMEs en la economía ecuatoriana. 
Aguirre Benalcázar, Martha Cecilia; Romero Hidalgo, Oscar Mauricio; Jaramillo Paredes, Marcia Fabiola y Ruiz Carrillo, Jonathan Alexander

Situación de la mediana empresa ecuatoriana: Rasgos distintivos de sus prácticas gerenciales

Sur Academia: Revista AcadémicaInvestigativa de la Facultad Juridica, Social y Administrativa, 4(8), 30-34.

Pérez, A. (2014). La comunicación interna en las Pymes. Capital Humano: Revista para la Integración y Desarrollo de los Recursos Humanos, 27(284), 8087.

Quiñónez, O., Castillo, S. N., Bruno, C. E., y Oyarvide, R. (2020). Gestión y comercialización: Pequeñas $\mathrm{y}$ medianas empresas de servicios en Ecuador. Revista de Ciencias Sociales (Ve), XXVI(3), 194-206. https://doi. org/10.31876/rcs.v26i3.33242

Rodríguez, I. (2020). Evaluación del auditor en la gestión financiera en las Pymes. Red Global de Conocimientos en Auditoria y Control Interno. https:// www.auditool.org/blog/auditoriaexterna/7096-evaluacion-del-auditoren-la-gestion-financiera-en-las-pymes

Rodríguez-Mendoza, R. L., y AvilesSotomayor, V. M. (2020). Las PYMES en Ecuador. Un análisis necesario. 593 Digital Publisher CEIT, 5(5-1), 191-200. https://doi.org/10.33386/593 dp.2020.5-1.337

Romero, J., Gutiérrez, J. M, Rodelo, H., Escorcia, T., Hernández, A., $\mathrm{y}$ Hernández, L. (2020). Conocimiento financiero de los micro y pequeños empresarios del sector porcino del Departamento del Atlántico Colombia. En V. Meriño, E. Martínez, A. Antúnez, J. Cruz, A. Pérez, L. Morán, H. Urzola, y M. Pérez (Eds.), Gestión del conocimiento. Perspectiva multidisciplinaria. Volumen 18, Colección Unión Global. (pp. 89108). Fondo Editorial Universitario de la Universidad Nacional Experimental Sur del Lago "Jesús María Semprún".

Romero, J., y Zabala, K. (2018). Planificación estratégica financiera en las cadenas de farmacias familiares del estado
Zulia - Venezuela. Revista de Ciencias Sociales (Ve), XXIV(4), 9-25.

Sanín, J. A., y Salanova, M. (2013). Satisfacción laboral: El camino entre el crecimiento psicológico y el desempeño laboral en empresas colombianas industriales y de servicios. Universitas Psychologica, 13(1). $\quad$ https://doi.org/10.11144/ Javeriana.UPSY13-1.slcp

Segredo, A. M., García, A. J., León, P., y Perdomo, I. (2017). Desarrollo organizacional, cultura organizacional y clima organizacional. Una aproximación conceptual. Revista de Información Cientifica para la Dirección en Salud. INFODIR, (24), $86-99$.

Sotomayor, J., Capa, L., y Ochoa, G. (2018). Gestión comercial y de mercadeo de las empresas de la provincia de El Oro. En L. Capa, J. Sotomayor y F. Vega (Coords.), La Provincia de El Oro algunas consideraciones de los sectores productivos y empresariales (pp. 101-117). Editorial UTMACH.

Thompson, A. A., Peteraf, M. A., Gamble, J. E., y Strickland, A. J. (2012). Administración estratégica: Teoría $y$ casos. Editorial McGraw-Hill/ Interamericana Editores.

Velásquez, Y., y Núñez, M. (2009). Los valores organizacionales presentes en la pyme metalmecánica venezolana. Un estudio comparado. COPÉRNICO, VI(12), 47-55.

Wang, H., Tong, L., Takeuchi, R., y George, G. (2016). Corporate Social Responsibility: An overview and new research directions. Academy of Management Journal, 59(2), 534-544. https://doi.org/10.5465/amj.2016.5001 